\title{
Molecular Simulation of Diffusion-controlled Kinetics in Stepwise Polymerization
}

\author{
Jing Liu, Yu Ma*, Rongliang Wu, Muhuo Yu* \\ State Key Laboratory for Modification of Chemical Fibers and Polymer Materials, College of \\ Materials Science and Engineering, Donghua University, Shanghai 201620, China
}

\begin{abstract}
The kinetics of stepwise polymerization in the diffusion-controlled regime was investigated using dynamic Monte Carlo simulation and scaling analysis. In analogy to Flory's expression, a concise expression of reaction kinetics in diffusion controlled regime was proposed, where the number average degree of polymerization was related to the initial concentration and reaction time through a power law dependence. In addition, the transition of kinetics from the classical reaction-controlled to diffusion-controlled regime was predicted and examined via simulation. The results provided a good description of kinetics for the late stage of stepwise polymerizations, and demonstrated good agreements with the various experiments.
\end{abstract}

Key words: reaction kinetics, stepwise polymerization, diffusion, scaling analysis, molecular simulation 


\section{Introduction}

Stepwise polymerization is one of the most important reaction in synthesis of a broad variety of polymer, including polyester, polyurethane, epoxy, and silicone etc. In stepwise polymerizations, any species (monomers, dimers, trimers, etc.) can react with each other in consecutive reactions, leading to an gradual increase in average molecular weight. Recently, Growing number of researches preparing linear molecules or aggregates through either condensation reaction[1,2], click reaction[3,4], molecular recognition[5] and other non-covalent forces[6], are in fact sharing the same kinetic mechanism

The pioneering work of Carothers in condensation polymerization led to the discovery of nylon and polyester over 85 years ago[7]. He elucidated the fundamental principles of stepwise polymerization with a second-order kinetic model. Afterwards, Flory[8] derived a quantitatively description of stepwise polymerization based on the equal reactivity assumption, as shown in Eq.1.

$$
\overline{X_{n}}=k c_{0} t+1
$$

where $\overline{X_{n}}$ represents for the number-average degree of polymerization, $c_{0}$ is the initial concentration of monomers, $k$ is the reaction rate constant related to the active energy or the reaction barrier[9]. The indicated linear increase of number average molecular weight with reaction time, however, has been demonstrated failed at high extent of reaction.

In fact, bias from second-order reaction kinetics was rather prevail in condensation polymerization. For example, several studies on the kinetics of synthesis of nylon 
indicated a third-order reaction kinetics when the extent of reaction was above $90 \%[10,11]$. So that a transition from second-order to third-order kinetics was suggested[12]. More recently, by comparing experimental data at low water concentration condition with theories, Zhang et al [13]suggested a kinetics between second-order and third-order in the later stages of polymerization. Similar deviation from second-order kinetics was also reported in the synthesis of poly (ethylene terephthalate). In the late stage of reaction, apparent rate constant decreased severely due to the increased distance between reactive end-groups, and diffusion was suggested to control the overall reaction rate[14,15].

Slowing down of the reaction rate due to diffusion could be more serious for the stepwise polymerization than normal second order reactions, since the polymer chains may extend till infinite length without termination. The reaction kinetics would eventually be controlled by diffusion if the time required for diffusion surpassed that for reaction[14]. Thus, the reactivity difference between molecules has only marginal effect on the reaction rates compared with the disparity of diffusion rate. In reality, the large scale production of commercial polyesters and nylon always meets the condition of diffusion control; in laboratory synthesis, diffusion affects the kinetics right after the starting of reaction for many cases, such as the synthesis of rigid rod polymers[16] For example, Cotts and Berry[16] showed that a prior parallel alignment was required for the reaction between two rigid rod like molecules. Thus, diffusion is decisive for the regulation of orientation[17], and shearing effectively accelerates the reaction through facilitating the diffusion[18]. This flow acceleration effect could also work 
for the synthesis of flexible polymers as predicted by Fredrickson and Leibler, provided the reaction system was controlled by diffusion[19]. In addition, the diffusion of polymer chains could also be effective in polymerizations under confinement or at interface, where the characteristic confinement dimension was smaller than the radius of gyration of the unconfined polymer[16,17].

Although the diffusion controlled regime has been realized for a long time, theoretical analysis and atomistic examination still face great challenges. First, till now, a concise expression like Eq.1 is still missing for the diffusion controlled regime, nor a model with appropriate approximations. Numerical polymerization kinetics could be obtained by solving an infinite series of derivative equations, but it is hardly useful in engineering designs. Moreover, these examinations of theoretical results were all based on numerical simulations without considering the real chain-like molecular architecture. Second, studies on the transition of reaction from the reaction-controlled regime to the diffusion-controlled regime are absent. These two competitive effects are not easily separated without a refined expression of apparent kinetics. Third, many side effects introduced by diffusion are still controversial. For example, in some literature, molecular weight distribution was suggested broader (polydispersity index PDI >2) in the diffusion controlled regime[18], and in other studies, it was narrower until less than 1.3 in the late stages[20].

The dynamic Monte Carlo (MC) simulation plays an important role in the understanding of polymerization processes for its well mimic of both chain architecture and its reptation motion. In present work, a concise expression of kinetics 
in the diffusion controlled regime is, for the first time, proposed and examined via dynamic Monte Carlo simulation in 3D lattice. The paper is organized as follow: we first introduced the simulation technique and verified the model in the reaction-controlled regime; then, based on the scaling arguments, we derived a simplified expression of kinetics in the diffusion-controlled regime and examined with the simulation; afterwards, the crossover from the reaction controlled to the diffusion controlled was studied theoretically and further verified by simulations; finally, we reported an intriguing odd-even effect incurred by the diffusion.

\section{Simulation techniques}

The thermodynamic and dynamic properties of polymers have been comprehensively studied via lattice model, for example on issues of phase separation[21], crystallization[22, 23] and chain dynamics[24-26], etc. The molecular simulation in the current study was performed in a $64^{3}$ cubic lattice box with periodic boundary conditions[27]. Generally, each monomer occupied a single lattice site, and a chain like polymer could be mimicked by consecutive occupied sites on cubic lattice linked with bonds. The distance between adjacent monomers along one chain was limited within one lattice unit, and the overlapping of monomer or crossing of bonds were rejected during the simulation in order to mimic the volume exclusion effect. In the current study, we focused only on the diffusion process under athermal condition, so that all kinds of inter-monomer interactions, except for the volume exclusion, were ignored. Before the simulation loop commences, a number of monomers each occupying a single site were placed randomly on the lattice. The concentration $c_{0}$ was 
defined as the ratio of initial inserted monomer and the total available sites. The rest empty sites played the role of athermal solvent monomers that allow the relaxation of polymer chains. All monomers were predefined as bifunctional, containing two reactive end groups $a$ and $b$, which could form bonds between each other upon reaction.

New configurations were generated via both monomer movements and reactions, and the algorithm was shown in flowchart of Figure 1. Either single monomers or monomers in polymers could relax by exchanging positions with randomly selected neighboring vacant sites with the restriction of volume exclusion. All trial moves with a single-site jumping model[28] were accepted unless violation of volume exclusion and breaking of bonds. Chain dynamics obtained following such relaxation algorithm have demonstrated good agreements with both the Rouse model in dilute solutions[29-31] and the reptation models in concentrated solutions[32,33]. Evolution time $t$ in the dynamic Monte Carlo simulation corresponds to the number of iterative simulation circles noted as Monte Carlo cycles (MCC), which is denoted as the attempted move of all monomers on average.

For the reaction part, in each Monte Carlo cycle, $N$ trial reactions were performed, where $N$ was the total number of monomers. In each trial, we randomly chose a monomer within the box, and a random neighbor sites of it, then check whether this two site could be linked with a new bond without violation of several critical rules. First, the two chosen sites should be both monomers at the end of chain. Second, the selected two monomers should contain opposite reactive group ( $a$ group react only 
with $b$ group). Third, In order to promote the formation of high molecular weight polymers, we forbade reactions within one molecule that resulting in cyclic molecules, and forbade chain transfers which might occur in real experiments, such as the synthesis of polyester[34]. Finally, the new bond should not introduce crossing with the exist bonds.

If all the above requirements were satisfied, whether a reaction could take place was then decided by comparing a random number $r \in[0,1)$ with reaction probability, $k_{M C}$. If $r<k_{M C}$, the reaction was allowed, otherwise, it was rejected. Similar reaction probability has been already successfully introduced in various reaction simulations[35-39]. For example, Yong et al.[36] introduced the reaction probability into dissipative particle dynamics to study the polymerization kinetics and gel formation. Liu and co-workers[35,39] introduced the reaction probability into molecular dynamics simulation to study the surface-initiated polymerization. Similar approach in the study of reaction kinetics has also been reported in the simulation of silico polymerization[38], bulk polymerization[40], and radical polymerization[41] and synthesis of hyperbranched polymer[42].

In our algorithm, we suggest the reaction probability $k_{M C}$ could be employed to study the kinetics of polymerization. According to Arrhenius equation[9], the apparent reaction rate is $k_{a p p}=A e^{-E a /(R T)}$, where $A$ is 'pre-exponential factor' or 'frequency factor', $E_{a}$ is the activation energy, $R$ is the gas constant, and $T$ is temperature. The pre-exponential factor $A$ was depended on collision frequency, reactive cross-section and steric effect in real reaction. In our simulation, $A$ was 
related to the reaction sampling frequency, lattice coordinate, and ratio of rejected sampling due to formation of cyclic chains etc. The exponential item $e^{-E a /(R T)}$, which was given by Boltzmann distribution, was the fraction of successful collisions that with the kinetic energy surpassed barrier energy $E_{a}$. In the simulation algorithm, the reaction probability $k_{M C}$ was defined as the acceptable ratio of reaction, which was directly analogous to $e^{-E a /(R T)}$. Therefore, the reaction probability $k_{M C}$ should be proportional to the apparent reaction rate $k_{a p p}$, and could be used to control the rate of reaction. In analogy to the equal reactivity assumption that the reaction rate coefficient was independent with chain length, we adopt a constant reaction probablity $k_{M C}$ throughout one simulation.

According to the proposed algorithm, diffusion could be then a separated factor comparing with local reaction possibility $k_{M C}$, because the reaction itself merely occurred between neighboring monomers or chain ends in current algorithm. Consequently, apparent reaction rate was affected by both $k_{M C}$ and diffusion rate of the chain ends, since two chain ends had to be transported firstly to nearby sites before reaction could occur. Thus, the diffusion process could also be a reaction rate determining step if the chain ends were sparsely populated, for example at the late stage of reaction. Systematically varying $k_{M C}$ allowed us to investigate the apparent reaction kinetics of both reaction controlled (early stage) and diffusion controlled (later stage) polymerizations by simply monitoring the growth rate of average chain length. 


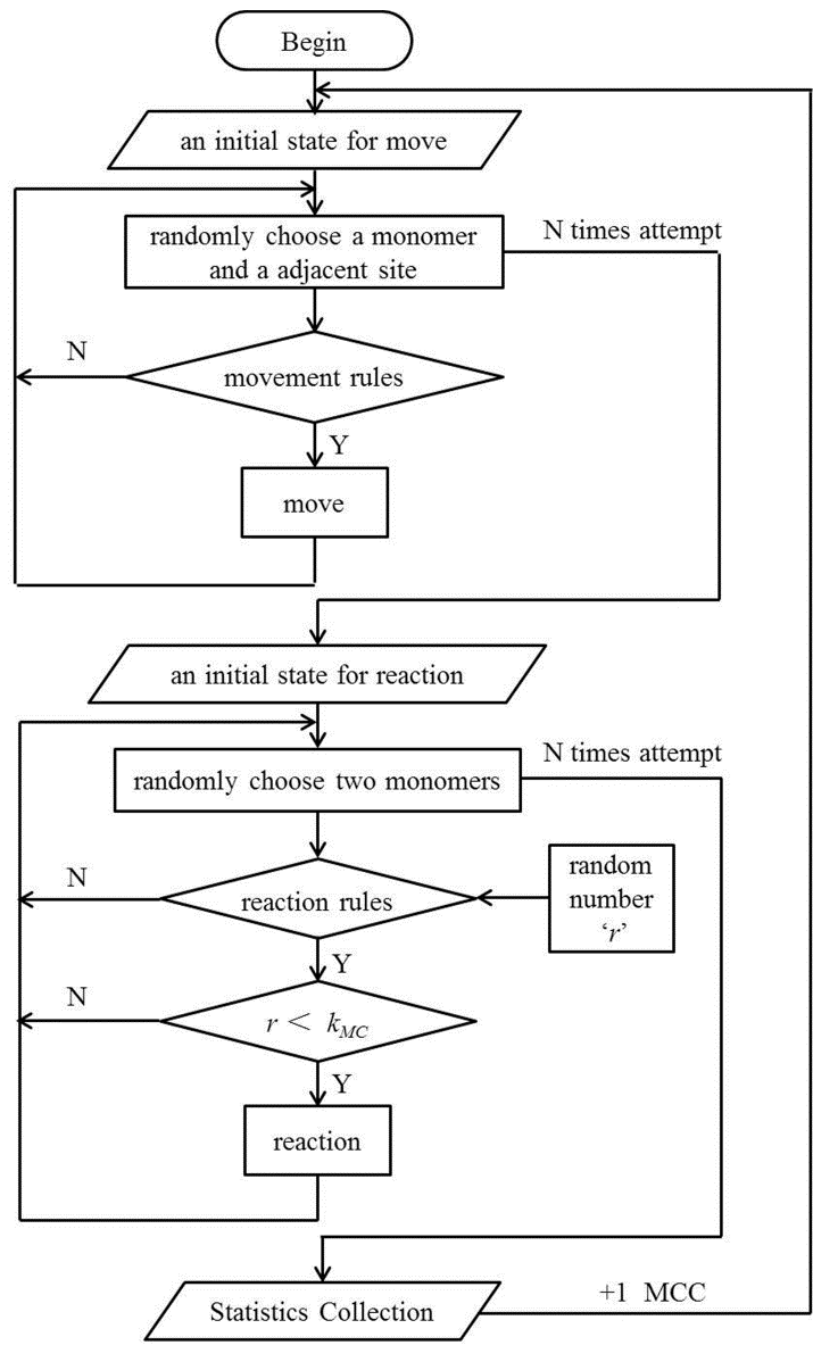

Figure 1. Flowchart of the move and reaction scheme in the computational model of stepwise polymerization. Details of the individual steps are described in the text.

In a typical simulation, as shown in Figure 2 and Figure 3, monomers were randomly placed in the lattice to prepare the initial states. Following the algorithm described above, monomers were almost immediately consumed, forming oligomers or short chains, while high molecular weight polymers emerged at high extent of reaction. The proposed simulation model could be easily extended to study reactions in more complex situations, for example, polymerization or copolymerization coupled with phase separation, crystallization, and systems involving nano confinement, shear flow, and chain stiffness etc. The proposed simulation model was independent on the 
entity of one monomer, and the type of reactive groups, thus it could also be suitable for the study of the particle assembling or supramolecular polymerization.

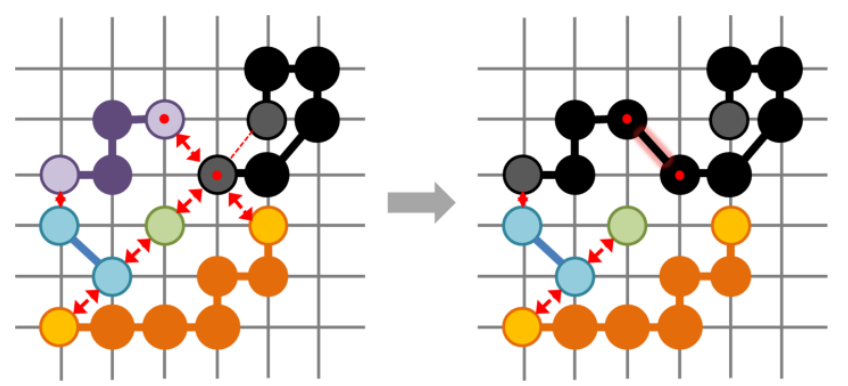

Figure 2. Schematic presentation of polymerization model. The functional groups of monomers or polymers can react with the surrounding groups to form a longer molecular chain. The solid lines represent monomers connected by solid bonds, the dashed lines are new bond positions produced in a step of model evolution, and the arrows present the reaction probability of monomer during the polymerization process.

(a)

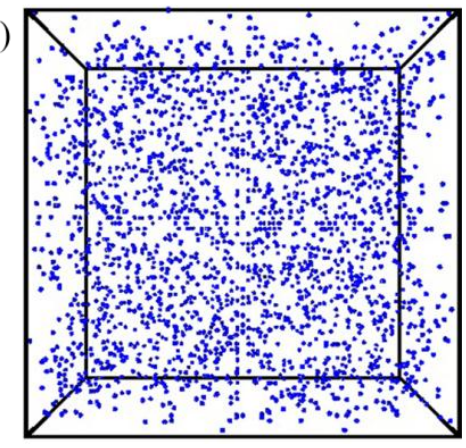

(b)

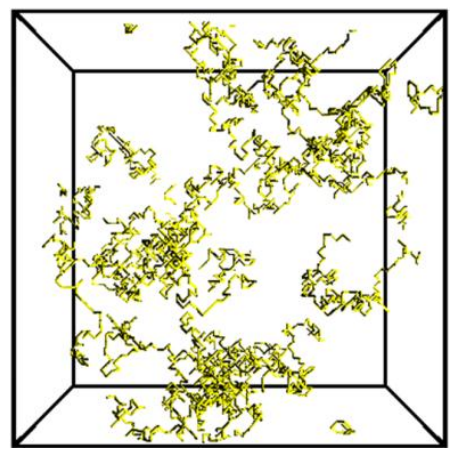

Figure 3. Snapshot of the stepwise polymerization process from (a) disordered monomers (blue) to (b) disordered polymer (yellow). The initial concentration $c_{0}=0.01$, and reaction rate constant $k_{M C}=0.001$. All the bonds are drawn in cylinders. 


\section{Results and discussion}

\subsection{Regime of reaction controlled polymerization}

The stepwise polymerization of monomers with different reactive end-groups, $a$ and $b$, was represented in Figure 4. Bonds could be formed between different species of group, $a-b$, while the reaction between the same species, namely the linkage between $a$ and $a$ or $b$ and $b$ was prohibited. Thus, the stoichiometric requirement for stepwise polymerization was always satisfied.

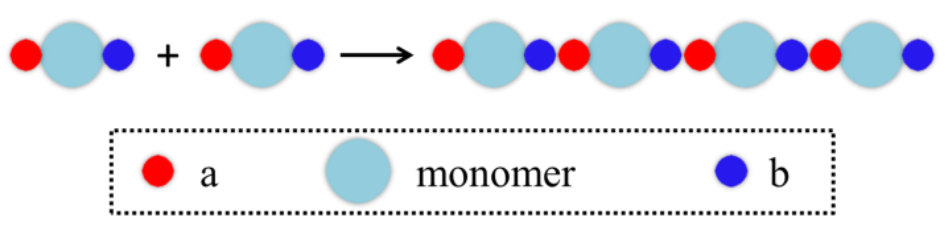

Figure 4. Schematic presentation of stepwise polymerization process of monomers with different species end groups. The red ball represented a-group. The blue ball represented b-group. The light ball represented monomer.

At first, we verified our simulation model by studying the polymerization with a small reaction probability $\left(k_{M C}=0.01\right)$. The simulation results of chain length $(x)$ distribution with respect to both number $\left(N_{x} / N\right)$ and weight $\left(W_{x} / W\right)$ at different extent of reaction $(p)$ were summarized in Figure 5. The data demonstrated a good agreement with the Flory's distribution given by Eq.2 and Eq.3[8,43], except for those of very short chains due to our irreversible reaction process and the inhibition of cyclization in simulation. Therefore, we suggested the algorithm that randomly chose neighboring reactive groups in simulation, which well mimicked the random reactions between groups in stepwise polymerization.

$\frac{N_{x}}{N}=\frac{N_{0} P_{x}}{x N}=(1-p) p^{x-1}$ 
$\frac{W_{x}}{W}=x(1-p)^{2} p^{x-1}$
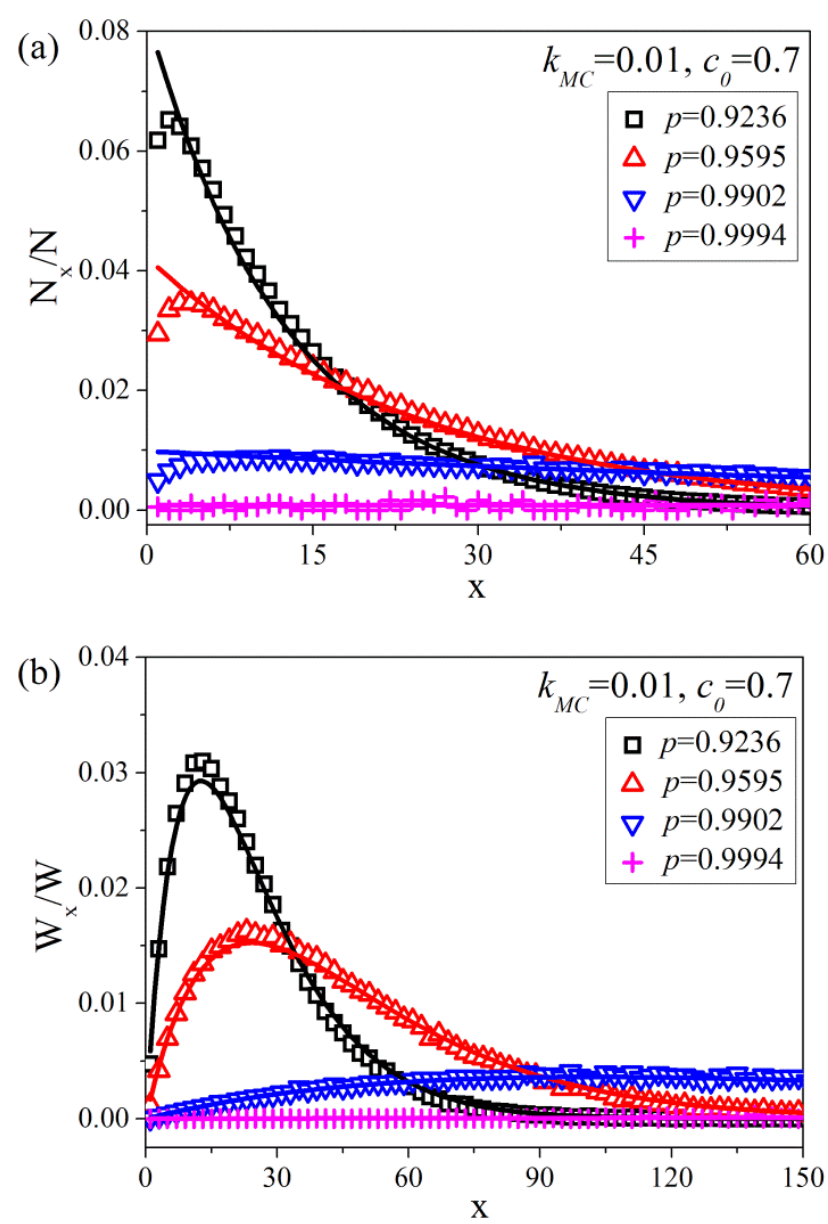

Figure 5. Number (a) and weight (b) average molecular weight distribution $\left(N_{X} / N\right.$, $W_{x} / W$ ) of $x$ at different extent of reaction $(p)$ in stepwise polymerization process, $k_{M C}=0.01, c_{0}=0.7$. The solid lines represented theoretical value calculated from Eq.2 and Eq.3. Each data point was average of result from fifty individual experiments.

As demonstrated in Figure 6, both the number-average degree of polymerization $\left(\overline{X_{n}}\right)$ and the weight-average degree of polymerization $\left(\overline{X_{w}}\right)$ increased linearly with the progress of polymerization at various concentrations, which showed a good correspondence with the second-order reaction kinetics as indicated by Eq.1. Apparent reaction rate could thus be determined by the slope of the kinetics curve in Figure 6b, which was suggested to be proportional to $k$ (or $k_{M C}$ in simulation) and the initial 
molecule concentration $c_{0}$ as indicated by Eq.1. The concentration dependence of apparent reaction rate was further confirmed in Figure $6 \mathrm{~b}$. At the same reaction time, the $\overline{X_{n}}$ achieved at high concentration $\left(c_{0}=0.7\right)$ was about 6 times higher than that in the dilute system $\left(c_{0}=0.1\right)$ because of the higher density of monomers. Thus, with properly defined reaction algorithm, we could quantitatively reproduce the kinetics of stepwise polymerization using the dynamic Monte Carlo simulation. The polydispersity index $(P D I)$ defined by the ratio of $\overline{X_{w}}$ and $\overline{X_{n}}$ exhibited a sudden increase right after the reaction had started, which indicated diversity of molecular weight appeared even at the initial stage of polymerization. The PDI in our studies went through a maximum at intermediate extent of reaction and reached a final value of approximately 1.9 as shown in Figure 6(a). According to the statistical theory[44], the PDI of polymer without regulator should be $\sim 2$ at high extent of reaction. The discrepancy was mainly caused by the small simulation volume compared with experiments, and the number of polymer chains in simulation was rather limited at high extent of reaction. Ultimately, there was only one chain left, and the PDI was 1, as suggested by Kéki and co-workers[45].

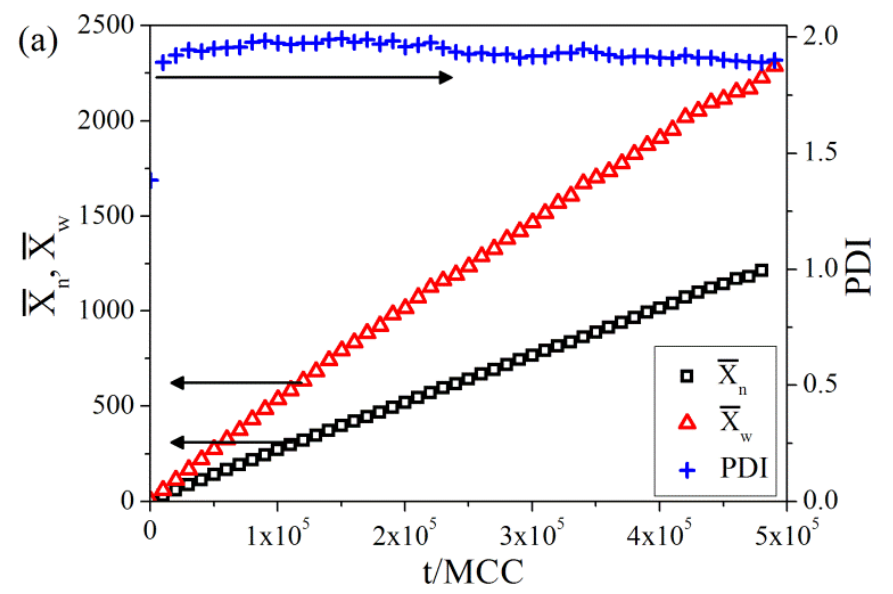




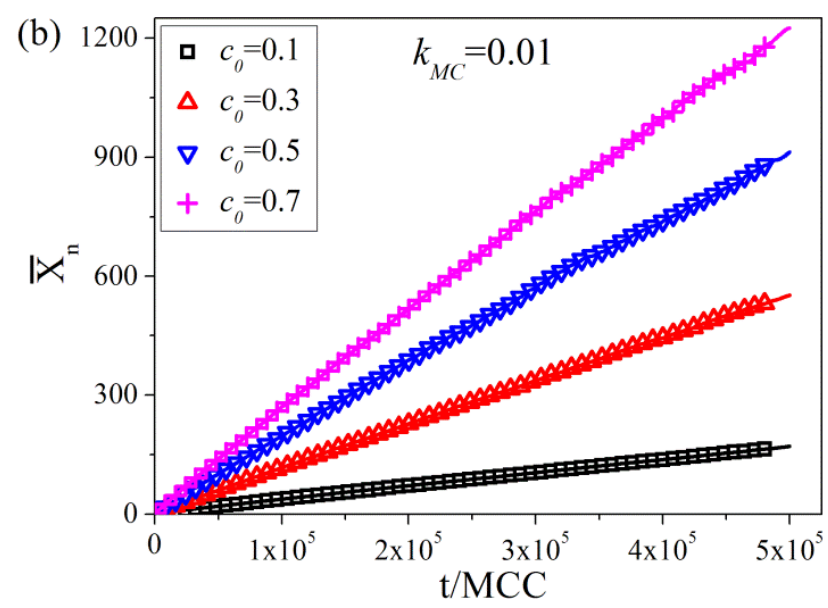

Figure 6. (a) Time evolution of $\overline{X_{n}}(\square), \overline{X_{w}}(\triangle)$ and $\operatorname{PDI}(+)$ at $k_{M C}=0.01$ and $c_{0}=0.7$. (b) The linear relationships between $\overline{X_{n}}$ and $\mathrm{t}$ in various concentrations. The data of $c_{0}=0.1(\square), 0.3(\triangle), 0.5(\nabla), 0.7(+)$ were averaged on the basis of thirty individual experiments. The solid lines were calculated from Eq.1.

We summarized the apparent reaction kinetics, noted as $k_{a p p}$, of various $k_{M C}$ by measuring the slope of $\overline{X_{n}}$ evolution across the range of three magnitudes in Figure 7. $k_{a p p} / c_{0}$ demonstrated a linear dependence with reaction probability $k_{M C}$ in logarithm plot, particularly in the lower regime $k_{M C}<0.1$, where the ratio of $k_{a p p} / c_{0}$ for all studied concentrations of the same $k_{M C}$ overlapped, indicating a well-defined second-order kinetics[46]. This is also a convincing demonstration that our simulation could quntatively study the polymerization kinetics. The ratio between $k_{a p p} / c_{0}$ and $k_{M C}$ depends on the simulation algorithm, such as the frequency of sampling, coordination number of the lattice, etc., and these values were invariant throughout all independent simulations with different $c_{0}$ or $k_{M C}$ [39]. At regime of high $k_{M C}>0.1$, an anomalous nonlinear behavior of $k_{a p p} / c_{0}$ with $k_{M C}$ and an extra dependence on $c_{0}$ deviating from the second-order kinetics was revealed. Severe slowing down of apparent reaction kinetics could be noticed in this regime, particularly at higher $c_{0}$, where diffusion 
became a non-negligible factor affecting the reaction at higher extent of reaction.

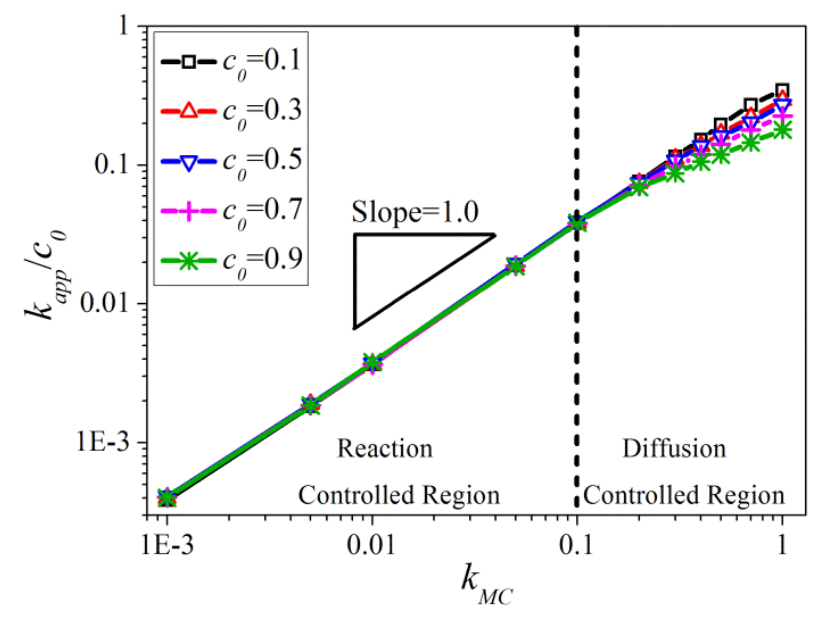

Figure 7. The relationship between $k_{M C}$ and $k_{a p p} / c_{0}$ in various concentrations, $c_{0}=0.1(\square), 0.3(\triangle), 0.5(\nabla), 0.7(+), 0.9(*)$ illustrated two different control factors during the polymerization process. The lines were drawn to guide the eyes. The linear relationships indicated a reaction-controlled mechanism, while non-linear parts were corresponding to diffusion-controlled mechanism.

Diffusion of long molecular chains had considerable effects on the reaction rate[47], particularly significant under viscous environment, or under strong confinements, etc.. Long molecular chains diffused over average a long distance to collide with each other before the chain reaction available[48]. As a result, other than the reaction barrier, apparent kinetics of reaction was mainly decided by time required for the end groups to encounter each other and the reaction rate slowly decreased with the increasing length of the molecular chain[49]. In case of the stepwise polymerization, at high extent of reaction, diffusion was severely slowed down due to the chain molecular structure and strong entanglements, as suggested by the Rouse model and reptation model. In addition, end groups were exhausted quickly at moderate extent of 
reaction, only one percent of reactive ends were left at the $p=0.99$, where $\overline{X_{n}}$ was only about 100. Combining the effect of slower chain dynamics and dilution of end groups, the reaction eventually entered a diffusion-controlled regime. Such transition was easily captured in our simulation when the reaction rate was relatively fast (relatively large $k_{M C}$ ) and the concentration was relatively high (relatively large $c_{0}$ ), and the diffusion effect could be eventually observed in a limited simulation window.

\subsection{Regime of diffusion-controlled polymerization}

The kinetics of stepwise polymerization is affected by two competing factors: the meeting of reactive chain ends which is controlled by diffusion, and the intrinsic rate of the polymeriztion process which is controlled by the chemistry. The slowest process decided the final apparent reaction kinetics, i.e. if the polymerization proceeded much solwer than diffusion, the system would be controlled by the reaction rate. On the other hand, if $k_{M C}$ became larger, the concentration of end groups would decrease rapidly, resulting in an increase of the mean distance between end groups, while the diffusion coefficient would decrease with increasing molecular weight. Since the reaction would occur only when two end groups met upon diffusion[50,51], and time required for diffusion overwhelm that of reaction, so finally diffusion would determine the apparent reaction kinetics.

Here, a rough scaling argument on the apparent reaction kinetics in the diffusion controlled regime was given and examined by our simulations. The scaling analysis of polymer structure and dynamics has achieved great success, owing to its simple mathematical derivation and accurate prediction of the properties related to the 
molecular weight. We supposed that the reactive chain ends were homogeneously distributed in $3 \mathrm{D}$ space, and the mean distance between the nearest reactive chain ends $(L)$ could be estimated by Eq.4.

$L=\left(\frac{\overline{X_{n}}}{c_{0}}\right)^{\frac{1}{3}}$

Even though $L$ should be smaller than the radius of chain, it was still good approximation to assume a Brownian motion of chain ends due to their faster relaxation rate comparing to the whole chain. Then, the time $t$ required for the meeting of two closest chain ends by diffusion was given in Eq.5.

$t=\frac{L^{2}}{6 D}$

In Eq. 5, $D$ represented the diffusion coefficient of chain ends, which has scaling relationship with both molecular weight and concentration[52]. Supposing the diffusion behavior of oligomer and polymer followed the Rouse model, $D$ could be expressed as Eq.6. Diffusion deviated from Rouse model was discussed in the latter part of this section.

$D \sim\left(\overline{X_{n}}\right)^{-1} c_{0}^{-1}$

Combining Eq.4-6, and neglecting the coefficients, we could obtain the scaling relationship between $\overline{X_{n}}$ and the time required for the meeting of nearest chain ends. If the reaction was faster than the diffusion of chain ends, the apparent reaction kinetics would be controlled solely by diffusion. Therefore, $\overline{X_{n}}$ in the diffusion-controlled regime could be expressed by Eq.7.

$\overline{X_{n}} \sim c_{0}^{-\frac{1}{5}} \cdot t^{\frac{3}{5}}$

In analogue to Eq.1, Eq.7 was a rather simplified expression. Here, several issues 
need to be discussed. First, unlike the reaction controlled regime where $\overline{X_{n}}$ and $\mathrm{t}$ showed a linear relationship in Eq.1, kinetics in the diffusion controlled regime demonstrated a power law dependence. Apparent reaction kinetics slowed down with time in the diffusion-controlled regime as indicated by the power index of 0.6 , which was also manifested by simulation in Figure 8. Coefficient in Eq. 7 was related to the particular reaction system or model, and omitted in scaling analyze. Second, in contrary to the positive linear dependence with $c_{0}$, the initial concentration dependence of $\overline{X_{n}}$ showed a small negative value -0.2 in the diffusion controlled regime. The apparent reaction kinetics was slowed down due to the low mobility of polymer in the concentrated and entangled environments. Third, $k_{M C}$ was not directly involved in the expression as the kinetics was controlled by diffusion instead of reaction. However, it could still be a secondary effect in this regime due to the different possibility for neighboring groups to react.

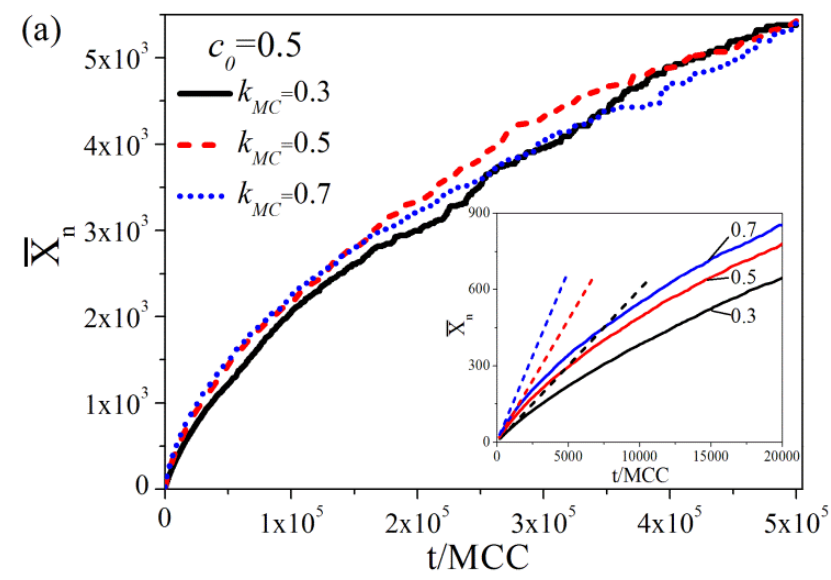



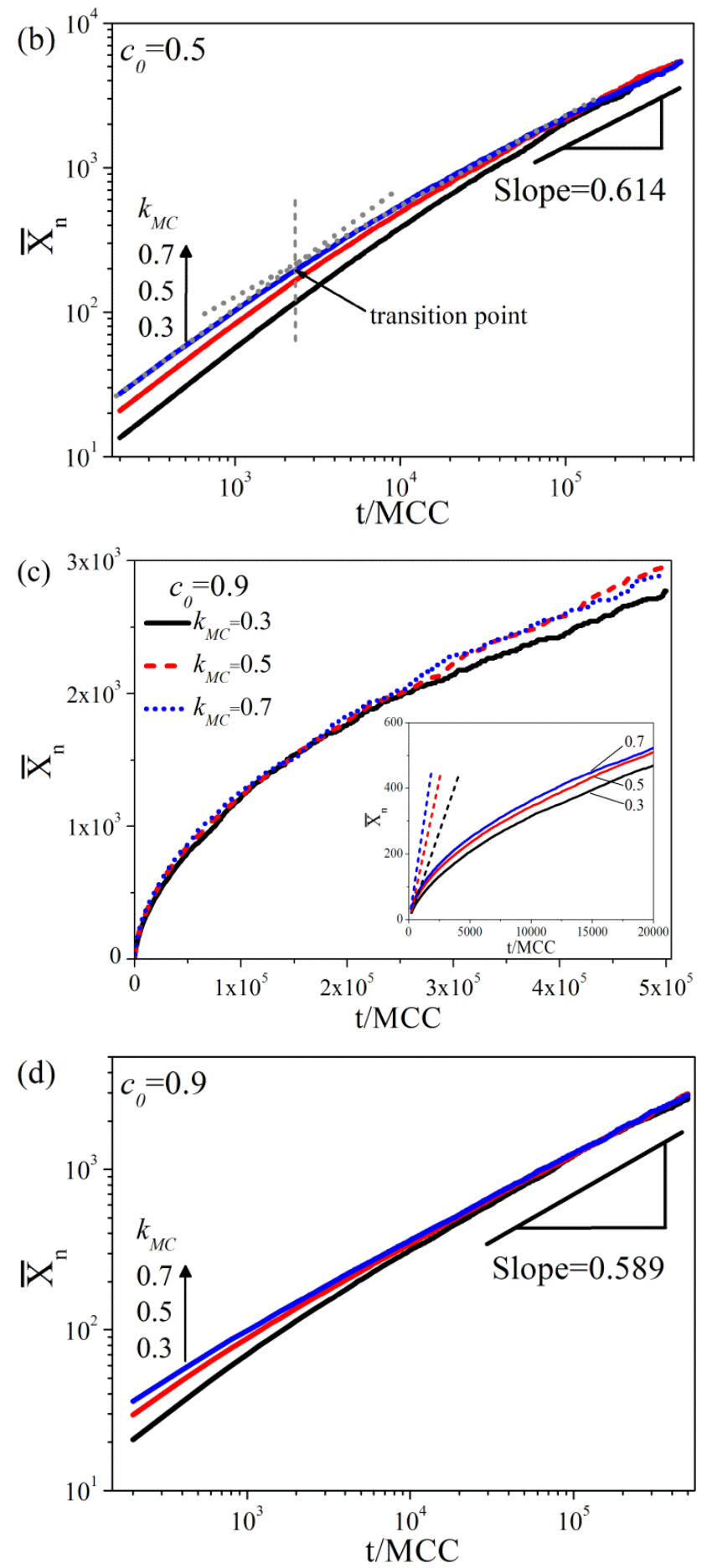

Figure 8. The time evolution of $\overline{X_{n}}$ during polymerization with different $k_{M C}=0.3(-), 0.5(--), 0.7(\bullet \bullet)$ at $c_{0}=0.5$ and $c_{0}=0.9$, plotted in linear scale (a), (c), and double logarithm scale (b), (d). Inserted figures in (a) and (c) showed the enlarged zone of the early stage, and the dashed lines inside represented the kinetics calculated based on Eq.1. Figures in (c) and (d) showed the scaling result of $\overline{X_{n}}$ and t which was entirely consistent with Eq.7. 
We traced the time evolution of $\overline{X_{n}}$ at relatively high $k_{M C}>0.1$ via simulation with the same algorithm. Other than the linear dependence in the reaction controlled regime (smaller $k_{M C}$ ), increasing of $\overline{X_{n}}$ was retarded and showed a typical power law relationship in the longer time range, as shown in Figure 8. With double logarithmic plots of $\overline{X_{n}}$ versus $t$ of different $k_{M C}$, we revealed the two regimes, i.e. the reaction-controlled at the early stage (slope $\approx 1$ ), and the diffusion controlled at the late stage (slope $\approx 0.6$ ). The scaling indicated from simulation demonstrated good agreement with previous theoretical analysis of Eq.7. As shown in the double logarithmic plots, the data lines with different $k_{M C}$ were separated with each other at early stage of polymerization. In this regime, reaction with higher $k_{M C}$ would achieve higher molecular weight. However, the lines merged with each other at later stage, indicating that effect of $k_{M C}$ was insignificant, and the apparent reaction kinetics was dominantly controlled by diffusion.

We summarized the scaling of $\overline{X_{n}}$ versus $t$ of various $k_{M C}$ and $c_{0}$ obtained from simulation in Figure 9. All scaling were in the range between 0.55 and 0.65 , which slightly deviated from the theoretical value 0.6. The deviation may be introduced due to the concentration dependence of the diffusion coefficient. According to the Zimm model and reptation model, the diffusion coefficient has the scaling of $D \sim\left(\overline{X_{n}}\right)^{-0.6}$ at lower concentration, and $D \sim\left(\overline{X_{n}}\right)^{-2} c_{0}^{-1.85}$ at higher concentration with entanglements. Thus, with the similar dedution mentioned above, the scaling exponent between $\overline{X_{n}}$ and reaction time $t$ should be 0.79 at lower concentration system and 0.38 at higher concentration system, which were both deviate severly from the scaling 
in Figure 9. In fact, in semi-dilute solution, chains were extensively interpenetrated, and hydrodynamic effects were sufficiently screened, particularly beyond the critical length. Meanwhile, considering the diffusion of chain ends was much faster than the whole chain, and the diffusion of shorter chain, which dominant the diffusion at late stage, were more likely Rouse type, we suggest the Rouse scaling in Eq. 6 could be a better approximation for current analyze. We should note that the derivation of Eq. 7 was rather oversimplified, for example without sufficient consideration of entanglement effect, and adopted an averaged diffusion coefficient instead of diffusion coefficient of individual chains of different length. However, since the error of scaling throughout the whole concentration range were less than 0.08 , we still suggest the Eq. 7 as a good approximation for this complicate situation, and could be adopted for the next step study.

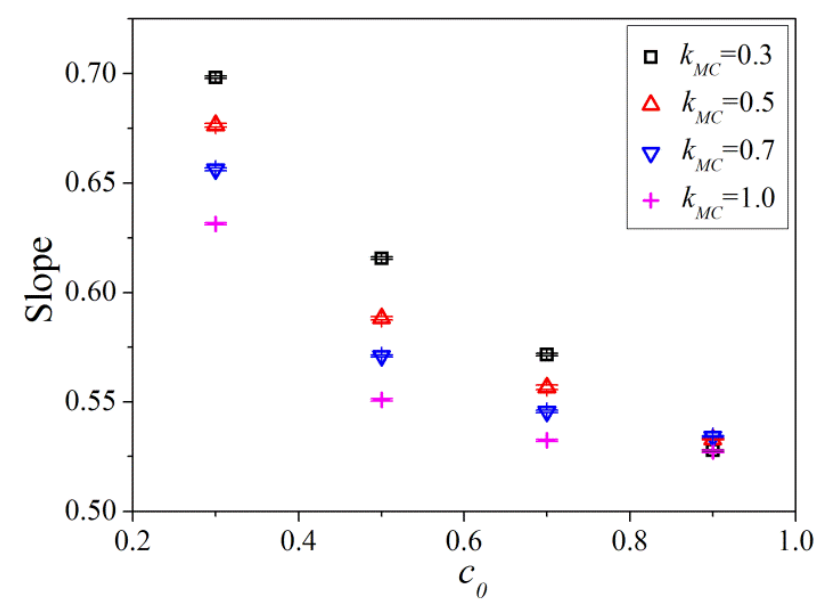

Figure 9. The scaling of kinetics of various $k_{M C}$ and $c_{0}$ in diffusion controlled regime. All data points were calculated by the slope of plots with averaged on the basis of ten individual experiments. Error bars are smaller than the symbol.

Similar scaling was also observed in late stage condensation polymerization or in 
solid state polymerization, and was treated previously as third-order reaction mechanism[53,54]. We suggest such transition could be partially attributed to the slow diffusion during reaction, which prevailed in stepwise polymerization. We speculated that such kind of transition could be much distinct for the synthesis of supramolecular polymers, where no small molecules were extracted, and moreover, their larger equilibrium constant was benefitted to the formation of very long chains.

Further examination of the diffusion-controlled reaction could be investigated from the $\overline{X_{n}}$ obtained at the same simulation time but at various concentration, as shown in Figure 10. Different from the increase of reaction rate with increasing $c_{0}$ in the reaction controlled regime, the reaction was slowed down in the diffusion controlled regime as a result of the lower diffusion coefficient at high concentration. Moreover, the scaling of concentration dependence was in good accordance with Eq.7 when $c_{0} \leqslant$ 0.7. However, at high concentration $\left(c_{0}>0.7\right)$, the Rouse dynamics failed because entanglements restricted the diffusion within a primitive tube. Hence, in practice, compromise of choosing proper $c_{0}$ had to be considered in order to synthesis ultra-high molecule weight polymer via stepwise polymerization. Synthesis in melt or concentrated solution would exhibit faster kinetics at the early stage, but the final $\overline{X_{n}}$ could be smaller due to the slow diffusion rate. 

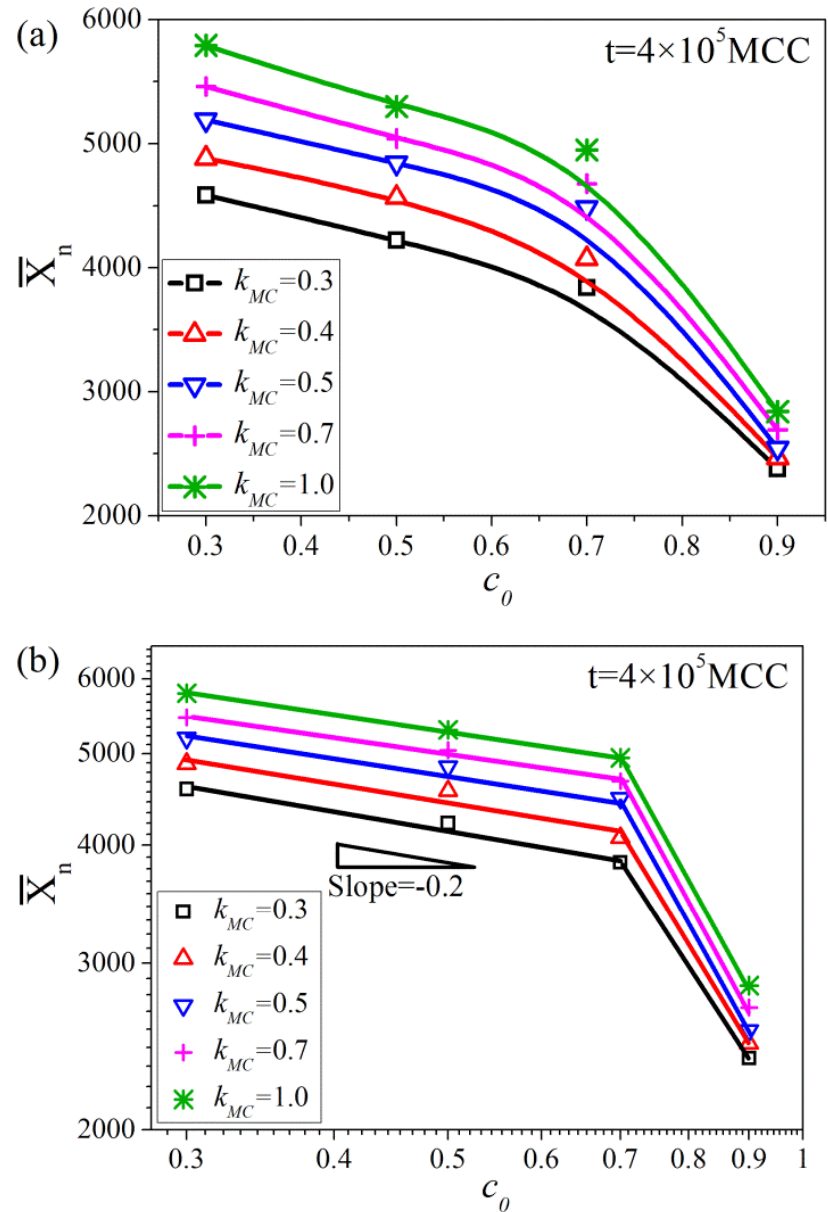

Figure 10. (a) The plots of $\overline{X_{n}}$ versus $c_{0}$ obtained at different $k_{M C}=0.3(\square), 0.4(\triangle)$, $0.5(\nabla), 0.7(+), 1.0(*)$. Sampling time was $\mathrm{t}=4 \times 10^{5} \mathrm{MCC}$ when the reaction system was controlled by diffusion. (b) Double logarithmic plots of $\overline{X_{n}}$ versus $c_{0}$ which are corresponding to the result in Figure 10(a). The lines are drawn to guide the eyes.

We further analyzed the transition from the reaction-controlled regime to the diffusion-controlled regime. We introduced a dimensionless number, $D_{a}$, which was denoted as the Damköhler number[55] and defined as the ratio of the diffusion timescale over the reaction timescale[56,57].

$D_{a}=\frac{\text { Time scale of diffusion event }}{\text { Time scale of reaction event }}=\frac{t_{\text {Diffusion }}}{t_{\text {Reaction }}}$

Combining Eq.1, 7, 8, the expression of $D_{a}$ is as follows:

$D_{a}=\frac{t_{\text {Diffusion }}}{t_{\text {Reaction }}} \sim\left(\overline{X_{n}}\right)^{\frac{2}{3}} \cdot c_{0} \frac{4}{3} \cdot k_{M C}$ 
$D_{a}$ represents the significance of diffusion in the reaction. $D_{a} \gg 1$ corresponds to the diffusion controlled regime, while $D_{a} \ll 1$ corresponds to the reaction controlled regime. By assigning $D_{a}=1$, we could obtain the transition point, ${\overline{X_{n}}}^{*}$ and $t^{*}$ as a function of $c_{0}$ and $k_{M C}$.

${\overline{X_{n}}}^{*} \sim c_{0}^{-2} \cdot k_{M C}^{-\frac{3}{2}}$

$t^{*} \sim c_{0}^{-3} \cdot k_{M C}^{-\frac{5}{2}}$

Both $\bar{X}_{n}^{*}$ and $t^{*}$ decreased with increasing $c_{0}$ and $k_{M C}$, and the dependence became stronger with increase of $c_{0}$. Such transition could be obtained in simulation by finding the crossover of extrapolated tangent line of the two scaling regimes in double logarithm plot, as shown in Figure 8(b). All anticipated scaling in Eq.10 and Eq.11 were quantitatively confirmed by our simulation as summarized in Figure 11 and Figure 12. The coincidence between simulation and scaling analyze may further demonstrate that Eq.7 was a good approximation of apparent kinetics in the diffusion controlled regime.

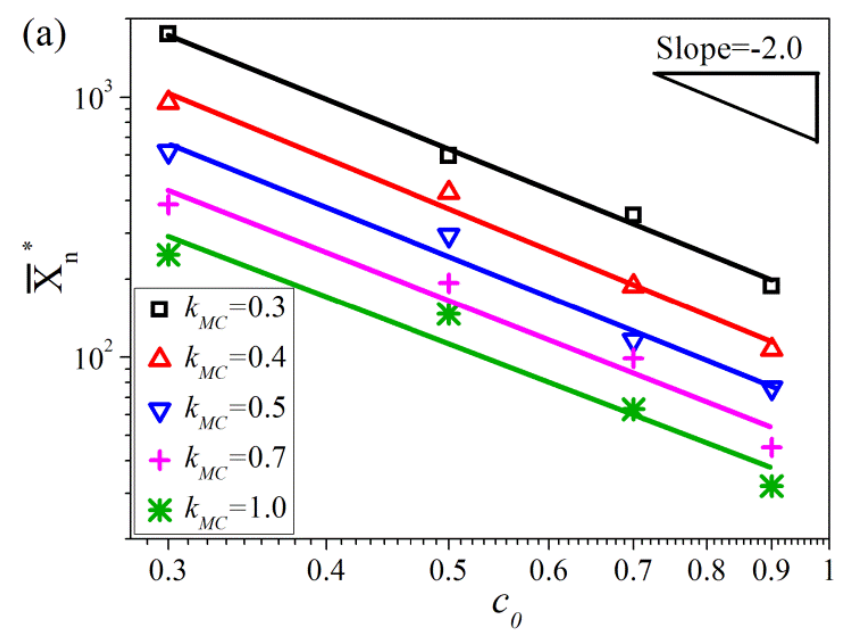




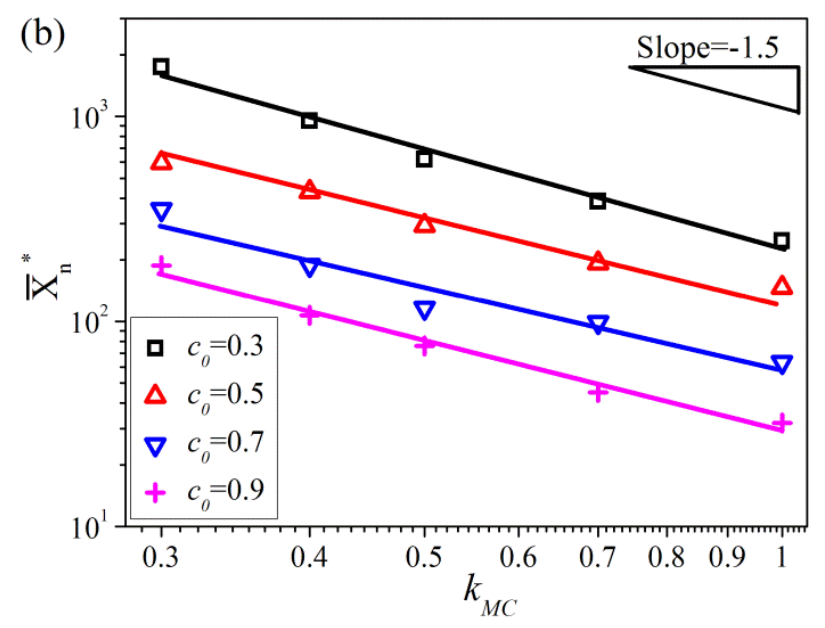

Figure 11. (a) Double logarithmic plots of $\overline{X_{n}^{*}}$ versus $c_{0}$ with different reaction rate $k_{M C}=0.3(\square), 0.4(\triangle), 0.5(\nabla), 0.7(+), 1.0(*)$. (b) Double logarithmic plots of $\overline{X_{n}^{*}}$ versus $k_{M C}$ with different initial concentration $c_{0}=0.3(\square), 0.5(\triangle), 0.7(\nabla), 0.9(+)$. The solid lines were drawn to guide the eyes. And slopes were denoted besides.
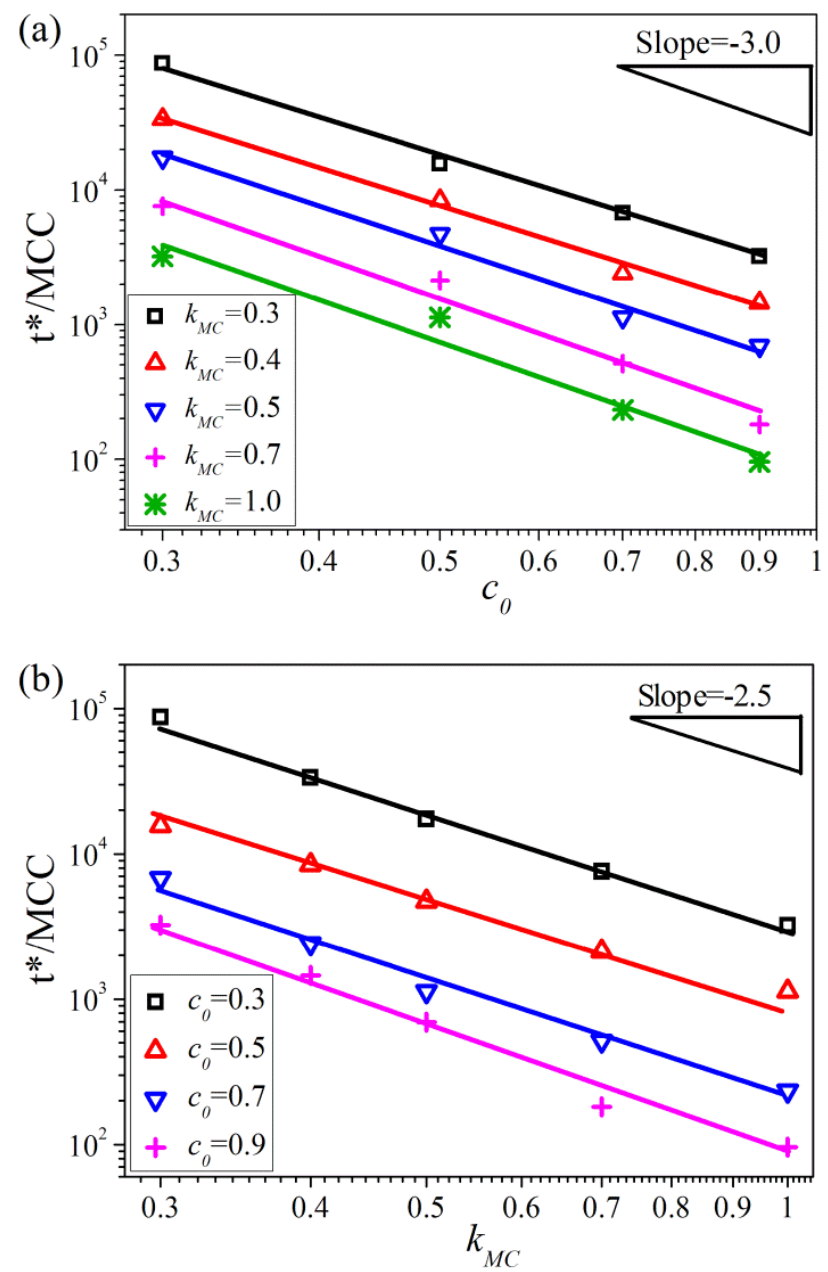
Figure 12. (a) Double logarithmic plots of $t^{*}$ versus $c_{0}$ with different reaction rate $k_{M C}=0.3(\square), 0.4(\triangle), 0.5(\nabla), 0.7(+), 1.0(*)$. (b) Double logarithmic plots of $t^{*}$ versus various $k_{M C}$ with different initial concentration $c_{0}=0.3(\square), 0.5(\triangle), 0.7(\nabla)$, $0.9(+)$.The solid lines were drawn to guide the eyes. And slopes were denoted besides.

In stepwise polymerization, the critical extent of reaction $\left(p^{*}\right)$ was frequently noted in experiments. Rozenberg et al.[58] investigated the kinetics of reactions of epoxy oligomers with amine, and discovered the diffusion controlled regime could only be found at extent of reaction higher than 0.98. Kline et al.[59] found that the controlling factor switched from kinetics to diffusion when the degree of polymerization was greater than 25 and the extent of reaction was greater than 0.923. Filgueiras[60] described the solid state polymerization of polyamide-6,6 with effective kinetic parameters under diffusion-controlled process, where the deviation from second order kinetics could be observed when $p>0.93$.

We derived the critical extent of reaction $\left(p^{*}\right)$ at the transition point based on the previous data shown in Figure 13. We noticed that in high concentration systems, the reaction entered the diffusion controlled regime at relatively low extent of reaction around 0.97 , corresponding to $\overline{X_{n}}$ being close to 30 . Our simulation results demonstrated good accordance with previously reported experiments. Furthermore, in dilute solution and with slow reaction kinetics, we could rarely find reaction controlled by diffusion, as the extent of reaction would approach one in such case. 

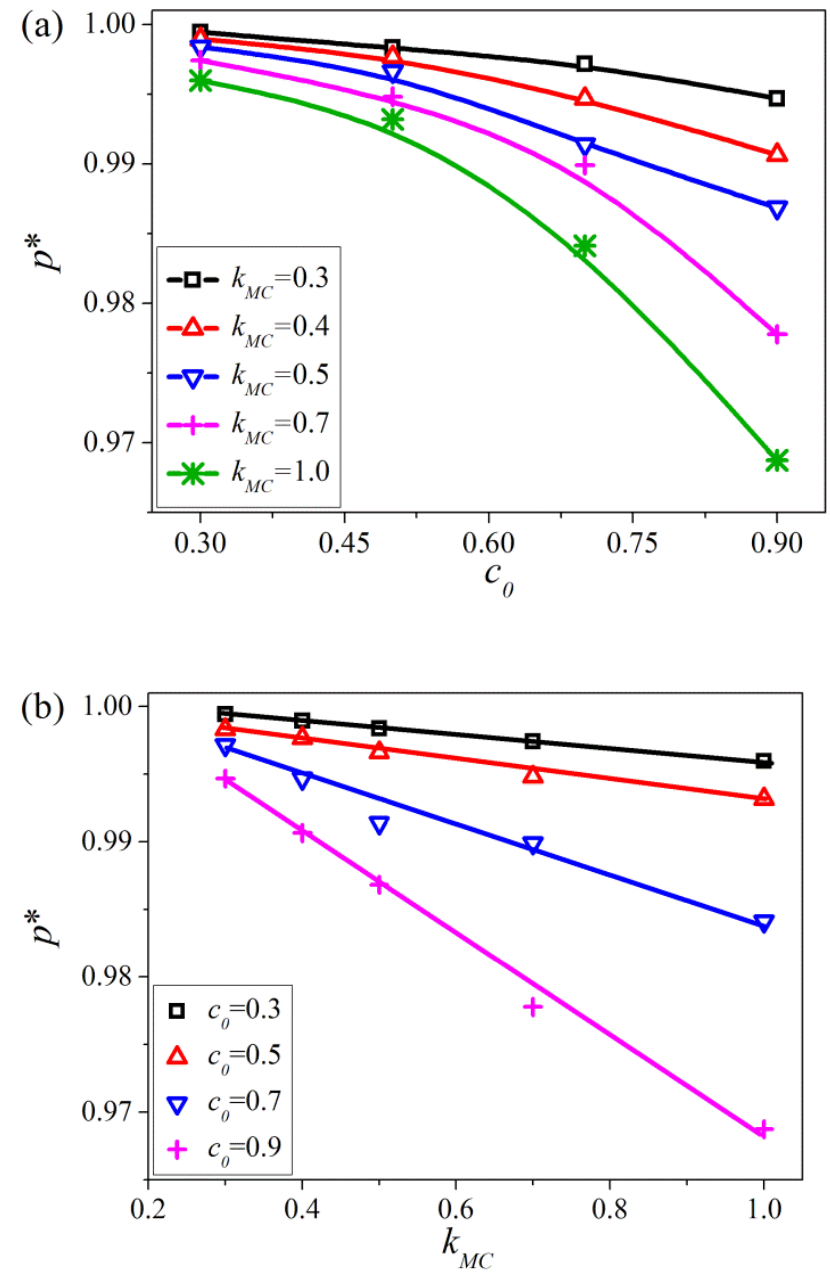

Figure 13. (a) The linear plots of $p^{*}$ versus $c_{0}$ with different reaction rate $k_{M C}=0.3(\square)$, $0.4(\triangle), 0.5(\nabla), 0.7(+), 1.0(*)$. (b) The linear plots of $p^{*}$ versus $k_{M C}$ with different initial concentration $c_{0}=0.3(\square), 0.5(\triangle), 0.7(\nabla), 0.9(+)$. The solid lines were drawn to guide the eyes.

Molecular weight distributions $\left(N_{x} / N, W_{x} / W\right)$ in the diffusion-controlled regime was further examined in Figure 14. The data indicated that the distribution of long chains were still in good coincident with Flory's distribution and not much affected by the diffusion process. But for shorter chain fraction $(\mathrm{x}<40)$, the deviation from Flory' s theoretical distribution could not be ignored. These results was definitely different from the distribution in Figure 5, which demonstrated a good agreement with the 
Flory' $\mathrm{s}$ distribution in reaction-controlled regime, even at high extent of reaction ( $p=0.9994)$. Furthermore, such deviation was more significant with increasing $c_{0}$, where diffusion was even retarded. In the diffusion-controlled regime, one chain end should react with another random end without previous know of its chain length. Therefore, all chain, more precisely all the long chain, would share the same possibility in reaction which satisfied the requirement of Flory's distribution, as exactly demonstrated in Figure 14. On the other hand, shorter chains has more opportunities and higher possibility to react due to their faster diffusion rate. And the rapid consumption of short chains may leaded to the lower fraction of short chain as observed in Figure 14. However, such deviation did not lead to big change in weight average distribution comparing with Flory's distribution, due to their minor contribution to the total weight, as shown in Fig 14(b). This was the reason that Flory's distribution was always valid in experiments, no matter whether diffusion dominant or not.

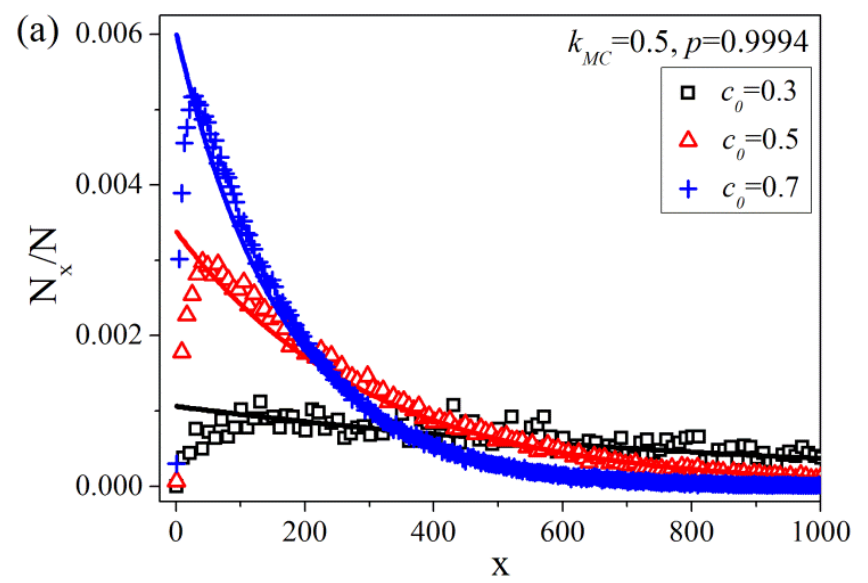




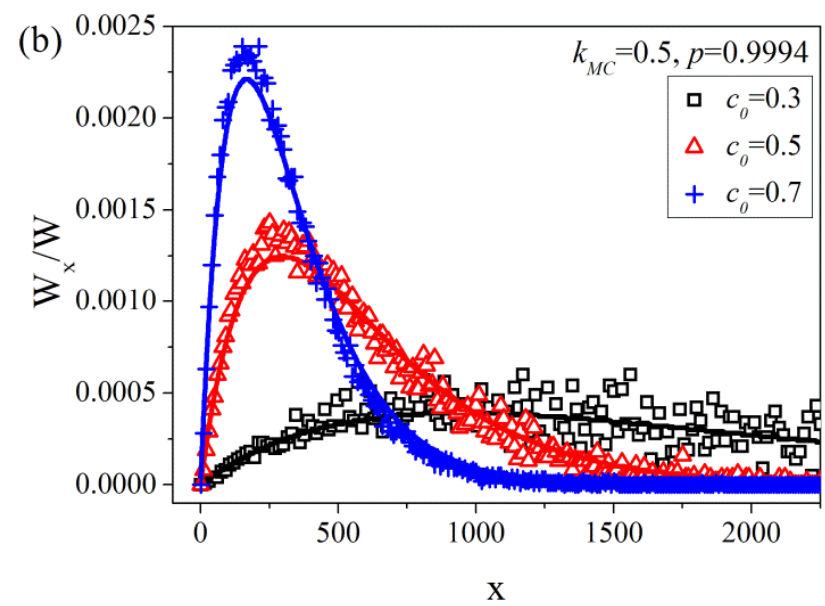

Figure 14. Number (a) and weight (b) average molecular weight distribution $\left(N_{X} / N\right.$, $\left.W_{x} / W\right)$ of $x$ at $p=0.9994$ in stepwise polymerization process with different initial concentration $c_{0}=0.3(\square), 0.5(\triangle), 0.7(+)$. The solid lines represented theoretical value calculated from Eq.2 and Eq.3. Each data point was average of result from three hundred individual experiments.

Finally, we consider the chain length distribution synthesized with equal amount of monomers with the same end groups, as illustrated in Figure 15.

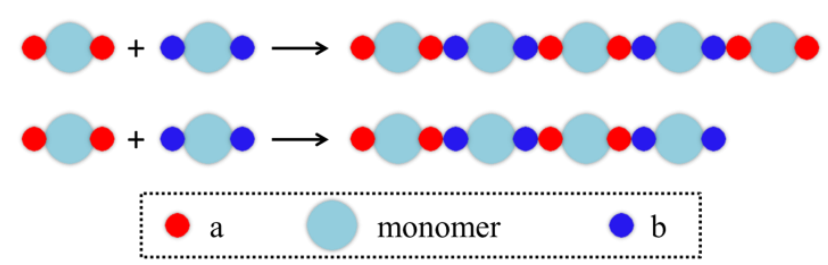

Figure 15. Schematic presentation of stepwise polymerization process of monomers with same end groups. The red ball represented a-group. The blue ball represented b-group. The light ball represented monomer. The polymer with odd number of molecules would have same end groups. While, the polymer with even number of molecules would have different end groups.

In Figure 16, an anomalous distribution could be observed if the reaction was controlled by diffusion. The fraction of polymer with odd number of molecules was much higher than the theory expected, however, the fraction of polymer with even 
number of molecules was much lower. Flory[61] reported a theoretical analysis of similar phenomena if the reactants were out of stoichiometric balance. However, we demonstrated in Figure 16, that these effects could also be reproduced even though the number of each kind of molecule was equal. And the discrepancy could be more evident at high $k_{M C}$ and initial concentration, when the diffusion effect was more significant. In reaction with $k_{M C}=0.01$, no odd-even effect was detected where the diffusion was faster than reaction, as shown in Figure 16(a). With the increasing reaction probability $\left(k_{M C}=0.1\right)$ and higher concentration $\left(c_{0}=0.7\right)$, the anomalous distribution could be very significant as shown in Figure 16(b) and Figure 16(c). And there were actually few chain with even number of monomers in final state if reaction was controlled by diffusion. We attributed this phenomenon to the uneven spatial distribution of each species of group due to the slow diffusion rate, even though initially they were homogeneous. Due to the faster consume of one species of end group than diffusion, a correlation hole between group $a$ and $b$ would be significant with progressing of polymerization which automatically generated $a$-rich domain and $b$-rich domain afterwards. Stoichiometric balance was failed in those domains and anomalous odd-even effect could be observed in such diffusion controlled reaction.

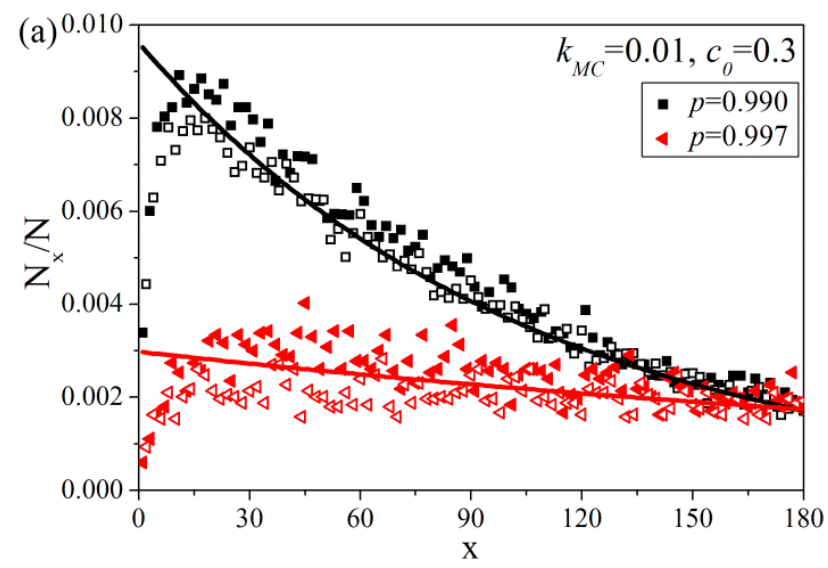



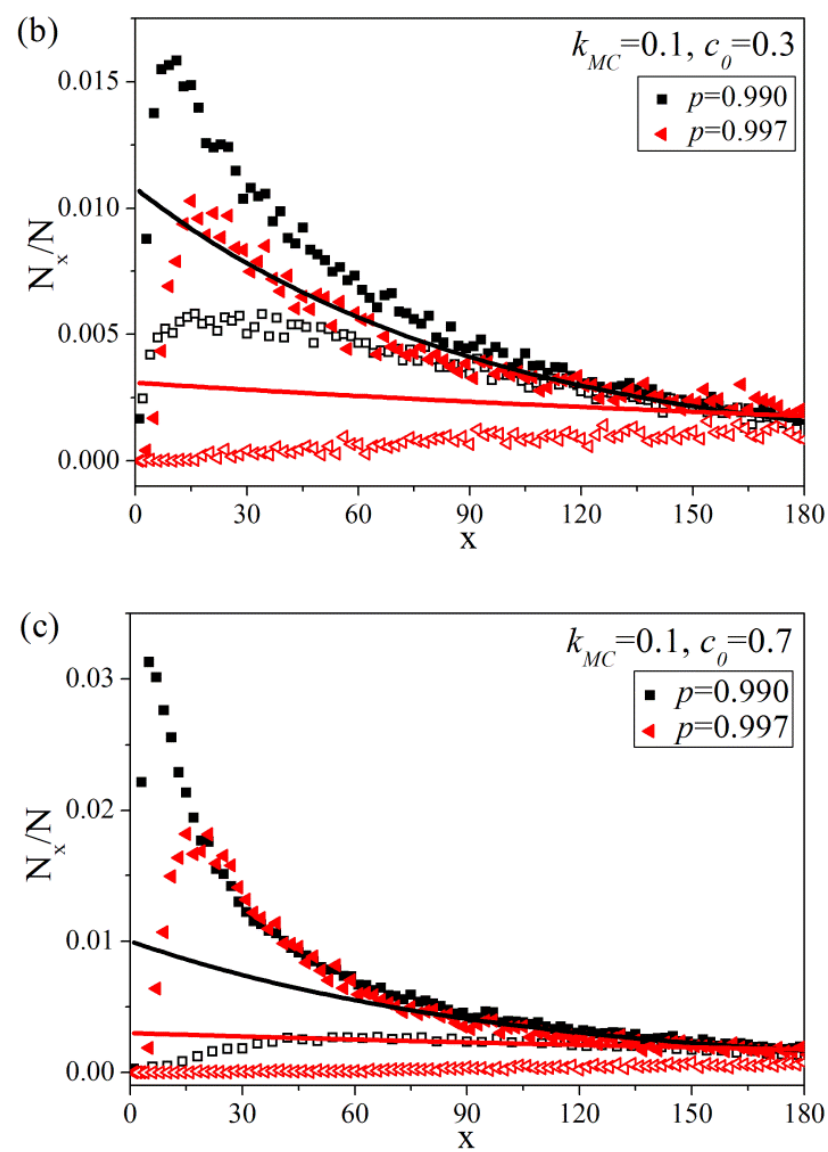

Figure 16. Number average molecular weight distribution $\left(N_{X} / N\right)$ of $x$ at different extent of reaction $(p)$ in stepwise polymerization process, (a) $k_{M C}=0.01, c_{0}=0.3$, (b) $k_{M C}=0.1, c_{0}=0.3$, (c) $k_{M C}=0.1, c_{0}=0.7$. The solid line presented the theoretical based on Eq.2. The extent of reaction of each curve was denoted, $p=0.990,0.997$. The solid symbols and the hollow symbols represented the population of polymer with the odd-number molecular chain and even-number molecular chain, respectively.

\section{Conclusion}

We have systematically investigated the apparent kinetics of stepwise polymerization in diffusion-controlled regime via dynamic Monte Carlo simulation. The new proposed simulation method has well captured the stepwise and stochastic essence of polymerization process, and demonstrated consistent data of kinetics with 
Flory's predictions in reaction controlled regime.

With increasing of the reaction rate and concentration, there was a crossover from reaction-controlled kinetics to diffusion-controlled kinetics, where the increasing of molecular weight followed roughly a scaling law that $\overline{X_{n}} \sim c_{0}^{-\frac{1}{5}} \cdot t^{\frac{3}{5}}$. Transition between the reaction-controlled regime and the diffusion-controlled regime was also

determined as ${\overline{X_{n}}}^{*} \sim c_{0}^{-2} \cdot k_{M C}^{-\frac{3}{2}}, t^{*} \sim c_{0}^{-3} \cdot k_{M C}^{-\frac{5}{2}}$, which showed a good agreements with simulation and with various experiment results of the polymerization of polyamide and epoxy. Meanwhile, an unexpected odd-even effect of molecule weight distribution was revealed in this regime. The simplified concise expression of kinetics could be applied in experiments control and engineering designs, particularly for those polymers synthesized or assembled in condensed or melt states.

\section{Acknowledgments}

This work was supported by the National Science Foundation of China (No. 21204011, No. 21304017), the National Basic Research Program of the China 973 Program (No. 2011CB606101), Fundamental Research Funds for the Central Universities, Innovation Program of Shanghai Science and Technology Commission (No. 14521100600) and the Innovation Program of Shanghai Municipal Education Commission. 


\section{References}

[1] K. Liu, Z. Nie, N. Zhao, W. Li, M. Rubinstein, E. Kumacheva, Step-growth polymerization of inorganic nanoparticles, science 329 (2010) 197-200.

[2] A.H. Gröschel, F.H. Schacher, H. Schmalz, O.V. Borisov, E.B. Zhulina, A. Walther, A.H. Müller, Precise hierarchical self-assembly of multicompartment micelles, Nature communications 3 (2012) 710.

[3] W.H. Binder, R. Sachsenhofer, 'Click'chemistry in polymer and materials science, Macromolecular Rapid Communications 28 (2007) 15-54.

[4] P.L. Golas, K. Matyjaszewski, Marrying click chemistry with polymerization: expanding the scope of polymeric materials, Chemical Society Reviews 39 (2010) 1338-1354.

[5] J. Stejskal, I. Sapurina, M. Trchová, Polyaniline nanostructures and the role of aniline oligomers in their formation, Progress in Polymer Science 35 (2010) $1420-1481$.

[6] A. Ciferri, Supramolecular polymerizations, Macromolecular rapid communications 23 (2002) 511-529.

[7] W.H. Carothers, Polymerization, Chemical Reviews 8 (1931) 353-426.

[8] P.J. Flory, Principles of polymer chemistry. Cornell University Press, Ithaca, 1953, pp. 69-105,317-346.

[9] P. Atkins, Physical Chemistry. Oxford University Press, New York, 2006, pp. 869-870.

[10] E. Roerdink, P. De Jong, J. Warnier, Study on the polycondensation kinetics of nylon-4,6 salt, Polymer communications 25 (1984) 194-195.

[11] E. Roerdink, J. Warnier, Preparation and properties of high molar mass nylon-4, 6: a new development in nylon polymers, Polymer 26 (1985) $1582-1588$.

[12] D. Steppan, M. Doherty, M. Malone, A kinetic and equilibrium model for nylon 6, 6 polymerization, Journal of applied polymer science 33 (1987) 2333-2344. 
[13] Zheng Wei , M.K. B., M.E.K. ., Y.K. Zhen, Effects of End-Group Balance on Melt-Phase Nylon 612 Polycondensation: Experimental Study and Mathematical Model, Ind. Eng. Chem. Res. 44 (2005) 2675-2686.

[14] J.D. Guzmán, R. Pollard, J.D. Schieber, Modeling of diffusion effects on step-growth polymerizations, Macromolecules 38 (2005) 188-195.

[15] S.A. Chen, F.L. Chen, Kinetics of polyesterification III: Solid-state polymerization of polyethylene terephthalate, Journal of Polymer Science Part A: Polymer Chemistry 25 (1987) 533-549.

[16] D.B. Cotts, G.C. Berry, Polymerization kinetics of rigid rodlike molecules: polycondensation of poly ([benzo (1,2-d:5,4-d') bisoxazole-2,6-diyl]-1, 4-phenylene), Macromolecules 14 (1981) 930-934.

[17] M. Doi, S.F. Edwards, The theory of polymer dynamics. Oxford University Press, New York, 1988, pp. 289-297.

[18] U. Agarwal, D. Khakhar, Enhancement of polymerization rates for rigid rod-like molecules by shearing, Nature 360 (1992) 53-55.

[19] G.H. Fredrickson, L. Leibler, Theory of diffusion-controlled reactions in polymers under flow, Macromolecules 29 (1996) 2674-2685.

[20] J.S. Gupta, A. Agge, D. Khakhar, Polymerization kinetics of rodlike molecules under quiescent conditions, AIChE journal 47 (2001) 177-186.

[21] W. Hu, V.B. Mathot, D. Frenkel, Phase transitions of bulk statistical copolymers studied by dynamic Monte Carlo simulations, Macromolecules 36 (2003) 2165-2175.

[22] G.I. Menon, R. Pandit, Crystallization and vitrification of semiflexible living polymers: A lattice model, Phys. Rev. E 59 (1999) 787.

[23] L. Toma, S. Toma, J.A. Subirana, Simulation of polymer crystallization through a dynamic Monte Carlo lattice model, Macromolecules 31 (1998) 2328-2334.

[24] C.C. Crabb, J. Kovac, Dynamics of cubic lattice models of polymer chains at high concentrations, Macromolecules 18 (1985) 1430-1435.

[25] P.H. Verdier, D.E. Kranbuehl, Simulation of polymer chain dynamics by lattice 
models with excluded volume: lattice dependence, Macromolecules 20 (1987) $1362-1368$.

[26] K. Haire, T. Carver, A. Windle, A Monte Carlo lattice model for chain diffusion in dense polymer systems and its interlocking with molecular dynamics simulation, Computational and Theoretical Polymer Science 11 (2001) 17-28.

[27] K. Kremer, K. Binder, Monte Carlo simulation of lattice models for macromolecules, Computer Physics Reports 7 (1988) 259-310.

[28] R. Larson, L. Scriven, H. Davis, Monte Carlo simulation of model amphiphile-oil-water systems, The Journal of chemical physics 83 (1985) 2411-2420.

[29] U. Agarwal, D. Khakhar, Diffusion-limited polymerization of rigid rodlike molecules: Dilute solutions, The Journal of chemical physics 96 (1992) $7125-7134$

[30] P.H. Verdier, W. Stockmayer, Monte Carlo calculations on the dynamics of polymers in dilute solution, The Journal of Chemical Physics 36 (1962) 227-235.

[31] H. Deutsch, K. Binder, Interdiffusion and self-diffusion in polymer mixtures: A Monte Carlo study, The Journal of chemical physics 94 (1991) 2294-2304

[32] W. Paul, K. Binder, D.W. Heermann, K. Kremer, Dynamics of polymer solutions and melts. Reptation predictions and scaling of relaxation times, The Journal of chemical physics 95 (1991) 7726-7740.

[33] G. Xu, J. Ding, Y. Yang, Monte Carlo simulation of self-avoiding lattice chains subject to simple shear flow. I. Model and simulation algorithm, The Journal of chemical physics 107 (1997) 4070-4084.

[34] M.S. Montaudo, Monte Carlo modeling of exchange reactions in polyesters. Dependence of copolymer composition on the exchange mechanism, Macromolecules 26 (1993) 2451-2454.

[35] H. Liu, M. Li, Z.-Y. Lu, Z.-G. Zhang, C.-C. Sun, T. Cui, Multiscale simulation study on the curing reaction and the network structure in a typical epoxy 
system, Macromolecules 44 (2011) 8650-8660.

[36] X. Yong, O. Kuksenok, A.C. Balazs, Modeling free radical polymerization using dissipative particle dynamics, Polymer 72 (2015) 217-225.

[37] D. Mukherji, C.F. Abrams, Mechanical behavior of highly cross-linked polymer networks and its links to microscopic structure, Physical Review E 79 (2009) 061802.

[38] J. Genzer, In silico polymerization: Computer simulation of controlled radical polymerization in bulk and on flat surfaces, Macromolecules 39 (2006) 7157-7169.

[39] H. Liu, M. Li, Z.-Y. Lu, Z.-G. Zhang, C.-C. Sun, Influence of surface-initiated polymerization rate and initiator density on the properties of polymer brushes, Macromolecules 42 (2009) 2863-2872.

[40] J. Ling, Y. Zhang, Z. Shen, J. Nie, Kinetics simulation of high viscous styrene bulk polymerization system, European polymer journal 37 (2001) 2407-2411.

[41] J. Ling, X. Ni, Y. Zhang, Z. Shen, Monte Carlo simulation of gas phase polymerization of 1, 3-butadiene Part I. Modeling and programming, Polymer 41 (2000) 8703-8707.

[42] L. Wang, X. He, Y. Chen, Diffusion-limited hyperbranched polymers with substitution effect, The Journal of chemical physics 134 (2011) 104901.

[43] P.J. Flory, Fundamental principles of condensation polymerization, Chemical Reviews 39 (1946) 137-197.

[44] H. Yu, N. Huang, C. Wang, Z. Tang, Modeling of poly (L-lactide) thermal degradation: Theoretical prediction of molecular weight and polydispersity index, Journal of applied polymer science 88 (2003) 2557-2562.

[45] S.n. Kéki, M.s. Zsuga, A.k. Kuki, Theoretical Size Distribution in Linear Step-Growth Polymerization for a Small Number of Reacting Species, The Journal of Physical Chemistry B 117 (2013) 4151-4155.

[46] J. He, H. Zhang, J. Chen, Y. Yang, Monte Carlo simulation of kinetics and chain length distributions in living free-radical polymerization, Macromolecules 30 (1997) 8010-8018. 
[47] L.T. Yan, B.H. Guo, J. Xu, X.M. Xie, Monte Carlo simulation of diffusion effects on chain-extension reactions, Journal of Polymer Science Part B: Polymer Physics 44 (2006) 2902-2911.

[48] W. Lu, J. Ding, Dynamic Monte Carlo simulation of polymerization of amphiphilic macromers in a selective solvent and associated chemical gelation, Macromolecules 39 (2006) 7433-7440.

[49] A.V. Berezkin, Y.V. Kudryavtsev, Simulation of End-Coupling Reactions at a Polymer-Polymer Interface: The Mechanism of Interfacial Roughness Development, Macromolecules 44 (2010) 112-121.

[50] K. Kang, S. Redner, Fluctuation-dominated kinetics in diffusion-controlled reactions, Physical Review A 32 (1985) 435-447.

[51] L.-T. Yan, B.-H. Guo, J. Xu, X.-M. Xie, Study diffusion effects on chain extension reactions based on the reptation theory, Polymer 47 (2006) 3696-3704.

[52] P. De Gennes, Kinetics of diffusion-controlled processes in dense polymer systems. II. Effects of entanglements, The Journal of Chemical Physics 76 (1982) 3322-3326.

[53] R. Srinivasan, C. Almonacil, S. Narayan, P. Desai, A. Abhiraman, Mechanism, kinetics and potential morphological consequences of solid-state polymerization, Macromolecules 31 (1998) 6813-6821.

[54] T. Kim, E. Lofgren, S. Jabarin, Solid-state polymerization of poly (ethylene terephthalate). I. Experimental study of the reaction kinetics and properties, Journal of applied polymer science 89 (2003) 197-212.

[55] K. Mayawala, D.G. Vlachos, J.S. Edwards, Spatial modeling of dimerization reaction dynamics in the plasma membrane: Monte Carlo vs. continuum differential equations, Biophysical chemistry 121 (2006) 194-208.

[56] H. Ouwersloot, J.V.-G. de Arellano, L. Ganzeveld, M. Krol, J. Lelieveld, The significance of species segregation for Amazonian chemistry. Joint session 8 of the 19th Symposium on Boundary Layers and Turbulence and the 29th Conference on Agricultural and Forest Meteorology, American Meteorological 
Society, 2-6 August 2010, Keystone, Colorado, USA, 2010. pp. J8. 1.

[57] M. Rubinstein, R.H. Colby, Polymer Physics. Oxford University Press, New York, 2003, pp. 309-312.

[58] B.A. Rozenberg, Kinetics, thermodynamics and mechanism of reactions of epoxy oligomers with amines, Advances in Polymer Science 75 (1986) 113-165.

[59] B.J. Kline, S.S. Lele, E.J. Beckman, A.J. Russell, Role of diffusion in biocatalytic polytransesterification, AIChE journal 47 (2001) 489-499.

[60] V. Filgueiras, S.N. Vouyiouka, M.O. Konstantakopoulou, A.C. Boussia, C.D. Papaspyrides, E.L. Lima, J.C. Pinto, Modeling of Polyamide 66 Solid State Polymerization: Drawing a Chemical Reaction Scheme, Macromolecular Reaction Engineering 9 (2015) 65-89.

[61] P.J. Flory, Molecular size distribution in linear condensation polymers, Journal of the American Chemical Society 58 (1936) 1877-1885. 


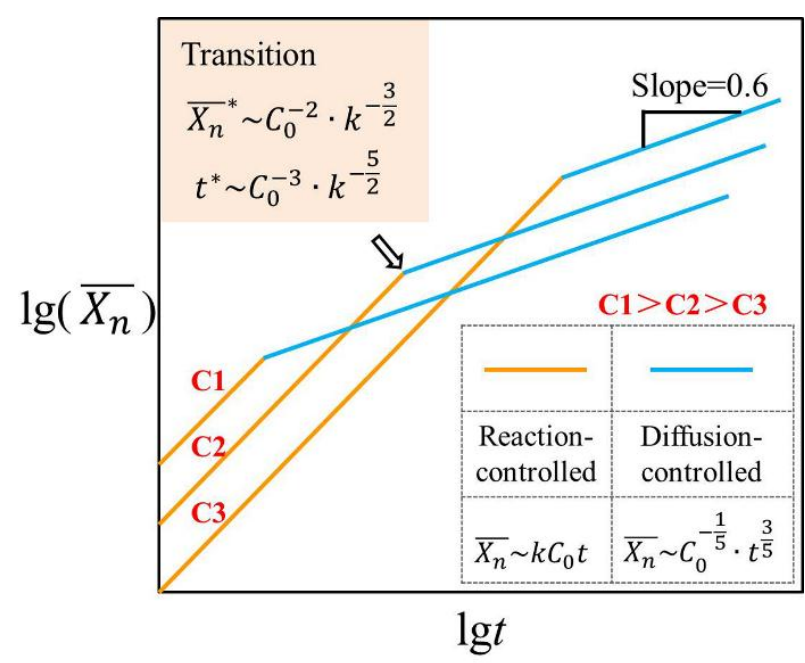

